\title{
Parâmetros ecológicos de fungos em Bromeliaceae em ecossistemas naturais e cultivadas na Bahia
}

\author{
Ecological parameters of fungi in Bromeliaceae \\ in natural and cultivated ecosystems in Bahia
}

\author{
Patrícia Martins Galvão Palha ${ }^{1,3}$, Jérsica dos Santos Silva ${ }^{1}$, Julielton Santos da Silva ${ }^{1}$, \\ Thaís Emanuelle Feijó de Lima ${ }^{1,2}$, Marcos Fábio Oliveira Marques ${ }^{2}$ \& José Luiz Bezerra ${ }^{1}$
}

\begin{abstract}
Resumo
Entre maio/2015 e fevereiro/2016 foi realizado um estudo objetivando estimar a riqueza, frequência e similaridade fúngica associada a Bromeliaceae de três áreas no estado da Bahia: Serra da Jibóia (município de Santa Terezinha), Morro da Redenção (município de Campo Formoso) e Orquilândia Tropical (município de Camaçari). Foram coletadas amostras de três espécies de Bromeliaceae por área: Alcantarea nahoumii, Vriesea bahiana, Aechmea sp. (Serra da Jibóia); Aechmea aquilega, Hohenbergia catingae, Tillandsia gardneri (Morro da Redenção) e Aechmea victoriana, A. naohoumii, Neoregelia compacta (Orquilândia Tropical). Foram selecionados dez indivíduos de cada espécie, destes, três folhas com sintomas ou sinais de fungos foram coletadas. Em laboratório efetuou-se o isolamento indireto dos fragmentos de folhas com desinfestação em álcool 70\%, NaOCl 1\% e lavagens em água destilada. Foram obtidos 19 gêneros distribuídos em 27 táxons. Na Orquilândia Tropical obteve-se a maior riqueza de espécies (17 táxons), as demais áreas apresentaram 14 táxons. Houve baixa similaridade fúngica nas plantas estudadas: 16,6\% a 50\% - Serra da Jibóia, 33,3\% a 58,8\% - Morro da Redenção e 23,3\% a 58,3\% - Orquilândia Tropical. Constatou-se diversidade fúngica para as espécies de bromélias tanto em ecossistemas naturais como em áreas cultivadas.
\end{abstract}

Palavras-chave: bromélias, microfungos, similaridade.

\begin{abstract}
Between May/2015 and February/2016 a study was carried out to estimate the richness, frequency and fungal similarity associated with Bromeliaceae from three areas in the Bahia state: Serra da Jibóia (municipality of Santa Terezinha), Morro da Redenção (municipality of Campo Formoso) and Orquilândia Tropical (municipality of Camaçari). Three species of Bromeliaceae were selected by area: Alcantarea nahoumii, Vriesea bahiana, Aechmea sp. (Serra da Jibóia); Aechmea aquilega, Hohenbergia catingae, Tillandsia gardneri (Morro da Redenção) and Aechmea Victoriana, A. naohoumii, Neoregelia compacta (Orquilândia Tropical). For each plant species 10 individuals were selected and three leaves of each with symptoms or fungal signs were collected. In the laboratory, indirect isolation was performed, which consisted of disinfestation in alcohol $70 \%, \mathrm{NaOCl} 1 \%$ and washing in distilled water of leaf fragments. A total richness of 19 genera distributed in 27 taxa was found in Orquilândia tropical the great species richness was obtained (17 taxa) and the other areas presented 14 taxa each. There was a low similarity between fungal communities in the studied plants: $16.6 \%$ to $50 \%$ - Serra da Jibóia, $33.3 \%$ to $58.8 \%$ - Morro da Redenção and $23.3 \%$ to $58.3 \%$ - Orquilândia Tropical. The study revealed a fungal diversity occuring in different species of bromeliads, both in natural ecosystems and in cultivated areas.
\end{abstract}

Key words: bromeliads, microfungi, similarity.

\footnotetext{
${ }^{1}$ Universidade Federal do Recôncavo da Bahia, R. Rui Barbosa, Lab. Microbiologia Agrícola, B1. L, 710, Centro, 44380-000, Cruz das Almas, BA, Brasil.

${ }^{2}$ Universidade do Estado da Bahia, Dep. Educação, Lab. Biologia Molecular e Fungos, Campus VII, Rod. Lomanto Júnior, BR-407, km 127, 48970-000, Senhor do Bonfim, BA, Brasil (In memoriam).

${ }^{3}$ Autor para correspondência: patypalha@gmail.com
} 


\section{Introdução}

Na Bahia destacam-se em importância dois biomas: a Caatinga, exclusivamente brasileira, com valores biológicos e econômicos, mas pouco conhecida (Loiola et al. 2012), e a Mata Atlântica, uma das áreas mais ricas, mas que vem sofrendo grande desmatamento, com cerca de $90 \%$ da sua vegetação nativa extinta pela ação antrópica (SOS Mata Atlântica \& Inpe 2013).

A Caatinga apresenta espécies vegetais xerófitas, herbáceas, cactáceas e bromeliáceas. Estas possuem espinhos e perdem suas folhas na estação seca (Araújo Filho \& Carvalho 1997). A Mata Atlântica, por sua vez, é composta por espécies de árvores nobres e de porte como canelas, imbuia, jacarandá, cedro, pau-brasil e outras, como também abrigam diversas espécies de animais (insetos, pássaros, mamíferos, anfíbios, entre outros). É um grande bioma em diversidade, contribuindo para que o Brasil seja um país rico em espécies de plantas e animais em relação a outros países (Brasil 2004). As bromélias na Mata Atlântica são epífitas em sua maioria, contribuem com recursos alimentares para vários seres vivos, são bioindicadoras e auxiliam na manutenção do microclima (Jensen et al. 2011). Na Caatinga também são encontradas bromélias endêmicas, de vários hábitos e que se adaptam ao ambiente xérico, em geral com folhas em rosetas gerando um tanque, com acúmulo de água e nutrientes favorecendo o equilíbrio ecológico desenvolvendo várias formas de vida (Islair et al. 2015).

Segundo Maia et al. (2015), com relação ao estudo de fungos no Brasil, a Mata Atlântica apresenta uma quantidade elevada de registros, com 3.017 espécies catalogadas, seguida pela Amazônia (1.050), Caatinga (999), Cerrado (638), Pampas (84) e Pantanal (35).

As bromeliáceas são plantas usadas com fins ornamentais de grande valor, com uma enorme variedade de cores e formas que as tornam admiráveis (Souza et al. 2015). Dessa forma, elas sofrem com a predação, assim torna-se essencial conhecer os fungos associados a essas espécies, visto que muitas são endêmicas e outras estão em risco de extinção. O presente estudo teve como objetivo estimar a riqueza, frequência e similaridade fúngica associada a bromélias em três áreas na Bahia (Mata Atlântica, Caatinga e Cultivada).

\section{Material e Métodos}

Seis coletas foram realizadas durante o período de maio de 2015 a fevereiro de 2016 , sendo duas em cada uma dos três municípios baianos: Campo Formoso (Morro da Redenção, $10^{\circ} 33$ '12,1'S, 40¹9'28,6”W) - região com clima predominante semiárido, inserido na Serra da Jacobina, pertencente à Chapada Diamantina, região de Caatinga (Ferreira 2013); Santa Terezinha (Serra da Jibóia, 1251'14,8'S, 39²8'36,4'W) localizada na porção leste da Bahia, no bioma Mata Atlântica com alta diversidade biológica (Blengini et al. 2015); e Camaçari - inserido no litoral norte do estado da Bahia (Orquilândia Tropical, $12^{\circ} 48^{\prime} 36^{\prime \prime} \mathrm{S}, 38^{\circ} 15^{\prime} 24^{\prime \prime} \mathrm{W}$ ) onde foram coletadas as espécies cultivadas.

Nas localidades selecionadas foram coletadas amostras de três espécies de Bromeliaceae de maior frequência nas áreas de estudo. De dez plantas de uma mesma espécie foram retiradas três folhas com sintomas ou sinais fúngicos, totalizando 30 folhas por espécie. No Morro da Redenção (Campo Formoso) foram coletadas as espécies: Aechmea aquilega (Salisb.) Griseb., Hohenbergia catingae Ule e Tillandsia gardneri Lindl. Na Serra da Jibóia (Santa Terezinha) Alcantarea nahoumii (Leme) J.R. Grant, Vriesea bahiana Leme e Aechmea sp. $\mathrm{Na}$ Orquilândia Tropical (Camaçari) Aechmea victoriana L.B. Sm, Alcantarea naohoumii (Leme) J.R. Grant e Neoregelia compacta (Mez) L.B. Sm.

As amostras coletadas das bromélias foram acondicionadas em sacos de papel kraft, identificadas e encaminhadas ao laboratório de Microbiologia Agrícola da Universidade Federal do Recôncavo da Bahia (UFRB). Fragmentos das folhas com lesões foram desinfestados em álcool $70 \%$ por dois minutos, em hipoclorito de sódio a $1 \%$ por dois minutos e lavados com água destilada por quatro vezes consecutivas. Após esse processo, os fragmentos foram transferidos para o meio de cultura Batata-dextrose-ágar (BDA) e incubados a temperatura ambiente. Os fungos, que cresceram ao redor desses fragmentos, foram repicados para obtenção de culturas puras, observados e caracterizados segundo características macro e micro-morfológicas. Alguns isolados fúngicos não produziram estruturas reprodutivas, mesmo em diferentes meios de cultivo, permaneceram na forma micelial e foram considerados Mycelia sterilia.

As culturas foram preservadas pelo método Castellani (1967) onde porções do micélio dos fungos foram colocadas em frasco tipo penicilina com água destilada esterilizada e fechadas.

As espécies foram identificadas utilizando a literatura especializada. Foram calculados índices 
ecológicos: riqueza, similaridade e frequência das espécies fúngicas. A riqueza foi determinada pelo número total de táxons encontrados nas áreas de coleta (Brower et al. 1998). A similaridade entre plantas da mesma área foi obtida a partir do coeficiente de Sørensen, $\mathrm{IS}=2 \mathrm{c} / \mathrm{A}+\mathrm{B} \times 100$, onde $\mathrm{c}=\mathrm{o}$ número de táxons comuns a duas espécies de plantas e $\mathrm{A}+\mathrm{B}=$ número de táxons presentes nas duas espécies vegetais (Muller-Dombois 1981). A frequência de ocorrência foi calculada com base na fórmula $\mathrm{F}=\mathrm{n} \times 100 / \mathrm{N}$, onde $\mathrm{n}=$ número de isolados para cada espécie fúngica e $\mathrm{N}=$ número total de isolados estudados das respectivas áreas pesquisadas (Dajoz 1983).

\section{Resultados e Discussão}

Foram obtidos 225 isolados fúngicos, destes isolados, 74 são provenientes das amostras de Morro da Redenção, 81 da Serra da Jibóia e 80 da Orquilândia Tropical (Tab. 1). A diferença encontrada entre as áreas quanto ao número de isolados fúngicos reflete os ambientes em que estão inseridas as bromélias, bem como as características vegetacionais e estruturais das plantas estudadas em cada área. A Serra da Jibóia apresenta morros com vegetações do tipo campo rupestres no topo, caatinga na base e mata higrófila nas encostas (Queiroz et al. 1996), apresentando clima subúmido a seco. As bromélias estudadas se encontravam em área de campo rupestre em solos rochosos e sujeitas a insolação, outra parte foi coletada na mata sob as árvores. O Morro da Redenção, por sua vez, inserido na Caatinga, apresenta plantas xerófilas, espinhentas, cactos e bromélias, a maioria rupícolas, arbustos e árvores de pequeno porte. O clima é caracterizado como semiárido a seco e úmido, a região faz parte do "Polígono das Secas" devido aos longos períodos de estiagem (Ferreira 2010). A Orquilândia Tropical possui um ambiente controlado para o cultivo de bromélias e orquídeas.

A frequência de ocorrência das populações fúngicas sobre cada planta por área possibilitou observar que todos os táxons estão classificados como pouco frequentes. Na Serra da Jibóia os valores variaram de 1,2 a 11,1\%, sendo as espécies mais comuns: Curvularia eragrostidis (Henn.) J.A. Mey. (6,2\%), Pestalotiopsis palmarum (Cooke) Steyaert, Purpureocillium lilacinum (Thom) Luangsa-ard, Houbraken, Hywel-Jones \& Samson (8,6\%) e Nigrospora oryzae (Berk. \& Broome) Petch $(11,1 \%)$. No Morro da Redenção, os valores foram de 1,4 a 10,8\%, com Acremonium murorum
(Corda) W. Gams e Hypoxylon sp. apresentando 5,4\%, Xylaria sp., 8,1\% e Trichoderma harzianum Rifai, $10,8 \%$ como os táxons mais comuns. Na área da Orquilândia Tropical verificou-se 1,3 a 12,5\% de frequência de ocorrência, com destaque para Phomopsis archeri B. Sutton, 6,3\%, Colletotrichum crassipes (Speg.) Arx, 11,3\% e N. oryzae, 12,5\% (Tab. 1). Os resultados demostram uma baixa frequência de espécies ocorrendo nos vegetais investigados, sendo $12,5 \%$ o máximo de frequência registrado para a espécie $N$. oryzae ocorrendo na bromélia Alcantarea nahoumii na Orquilândia Tropical. As folhas de bromélias apresentam estrutura rígida (Silva \& Scatena 2011) o que pode dificultar a penetração e colonização de fungos conforme foi observado nos ambientes estudados.

As espécies fúngicas mais encontradas para a Serra da Jibóia foram Nigrospora oryzae em Vriesea bahiana $(11,1 \%)$ e Alcantarea nahoumii (7,4\%), Purpureocillium lilacinum (8,6\%) e Curvularia eragrostidis (6,2\%) em Aechmea aquilega. No Morro da Rendenção, Trichoderma harzianum (10,8\%) foi a mais comum em Aechmea aquilega. Na Orquilândia Tropical foram mais comum Nigrospora oryzae em Alcantarea nahoumii (12,5\%), Colletotrichum crassipes (11,3\%) e Phomopsis archeri (6,3\%) em Aechmea victoriana. A espécie Nigrospora oryzae, que ocorreu em todas as bromélias e locais, apresenta crescimento rápido, podendo ocorrer como endófita, sapróbia ou patogênica dependendo das condições ambientais (Kumar et al. 2015).

A riqueza de espécies fúngicas nas áreas estudadas foi de 27 táxons, distribuídos em 19 gêneros, sendo 14 táxons para Serra da Jibóia (Santa Terezinha) e para Morro da Redenção (Campo Formoso) e 17 para Orquilândia Tropical (Camaçari) (Tab. 1). Dentre os locais estudados apenas cinco táxons foram comuns aos três: Aspergillus niger Tiegh., Colletotrichum gloeosporioides (Penz.) Penz \& Sacc., Nigrospora oryzae, Pestalotiopsis palmarum e Purpureocillium lilacinum. Orquilândia Tropical apresentou o maior número de táxons exclusivos, seguido pelo Morro da Redenção e Serra da Jibóia (Fig. 1).

Na Orquilândia Tropical a maior quantidade de espécies de fungos associados às bromélias pode ser justificada por ser um ambiente de estufa, com uma diversidade de plantas de vários ecossistemas e com controle de temperatura e umidade, sendo um ambiente confinado, o que concentra um grande número de esporos no ar. A maior parte das espécies encontradas nesse 


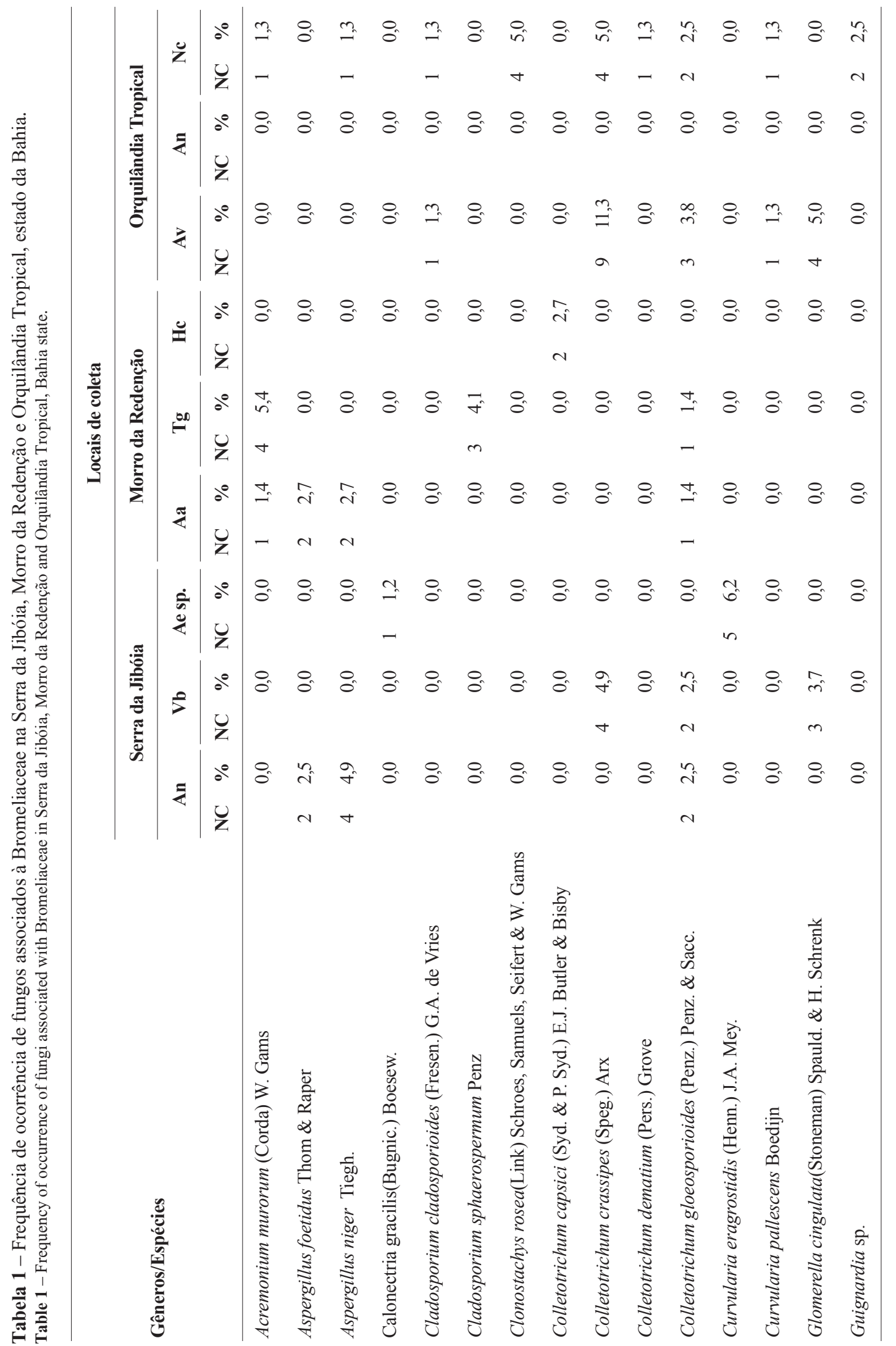




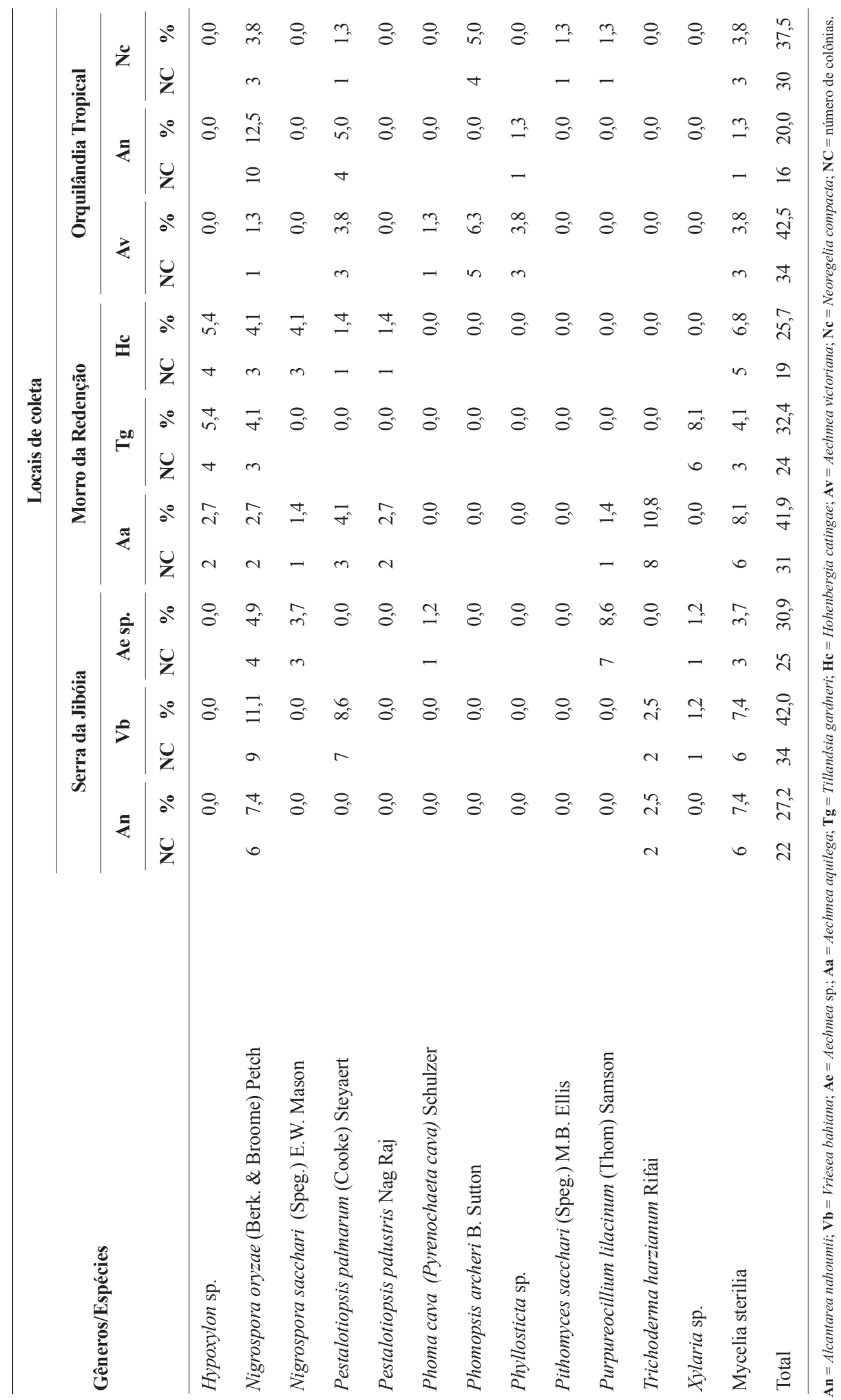




\section{Serra da Jibóia Morro da Redenção}

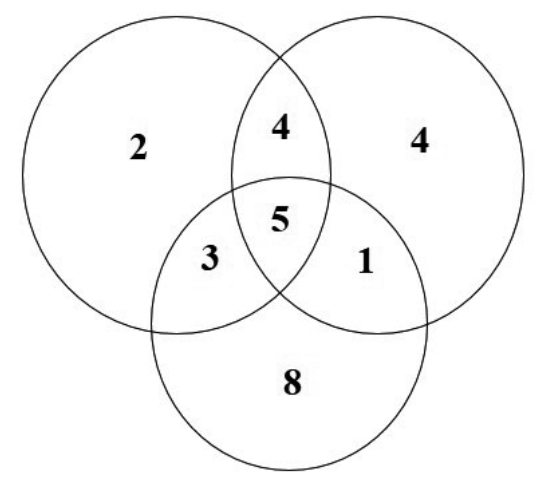

\section{Orquilândia Tropical}

Figura 1 - Táxons exclusivos e comuns em Serra da Jibóia (Santa Terezinha), Morro da Redenção (Campo Formoso) e Orquilândia Tropical (Camaçari), Bahia. Figure 1 - Exclusive and common taxa in Serra da Jibóia (Santa Terezinha), Morro da Redenção (Campo Formoso) and Tropical Orquilândia (Camaçari), Bahia.

espaço foi trazida da Tailândia. Vale salientar que em áreas cultivadas há aplicações de defensivos e adubos químicos, o que pode influenciar na nutrição das plantas e torná-las suscetíveis a pragas e doenças (Tokeshi 2002; Picanço 2010), bem como pode haver seleção em favor das espécies fúngicas resistentes aos produtos. Contudo na Orquilândia tropical foram registradas espécies comuns a Aechmea victoriana e Neoregelia compacta [Cladosporium cladosporioides (Fresen.) G.A. de Vries, Curvularia pallescens Boedijn, Phomopsis archeri], enquanto Aechmea victoriana e Alcantarea nahoumii tiveram apenas uma espécie fúngica em comum (Phyllosticta sp.). Clonostachys rosea (Link) Schroers, Samuels, Seifert \& W. Gams, Colletotrichum dematium (Pers.) Grove, Guignardia sp. e Pithomyces sacchari (Speg.) M.B. Ellis foram registrados apenas em Neoregelia compacta. Os táxons Guignardia sp. e Phyllosticta sp. são estágios sexuais do mesmo gênero de fungo, mas foram encontrados em hospedeiros diferentes e podem corresponder a duas espécies diferentes.

As cinco espécies de fungos comuns aos locais estudados são frequentemente encontradas em outros hospedeiros, sendo amplamente registradas (Costa et al. 2009; Reis et al. 2009; Costa et al. 2012; Mussi-Dias et al. 2012; Santos et al. 2014; Lima \& Cavalcanti 2014; Lima et al. 2014; Costa \& Gusmão 2016; Candeias et al. 2016).
Apesar das espécies de bromélias ocorrerem em ambientes distintos, a riqueza fúngica foi semelhante em todas as áreas. No entanto, a composição de espécies para cada área e espécie de bromélia revela-se heterogênea. Essa heterogeneidade tem sido encontrada em estudos de diversidade fúngica conduzidos na mata atlântica, comparando-se pontos de amostragem em uma mesma área e substratos diferentes (Marques et al. 2008). Na caatinga, Silva et al. (2014) estudando substratos vegetais submersos, obtiveram 90 espécies de fungos anamorfos demonstrando a riqueza de espécies fúngicas em diferentes substratos e condições de decomposição. Os dados encontrados nessa pesquisa confirmam a existência de uma comunidade fúngica característica associada a bromélias em ecossistemas naturais e cultivados.

As similaridades fúngicas entre as plantas ocorrentes em uma mesma área investigada foram baixas. Para a Serra da Jibóia a similaridade variou de $16,6 \%$ a $50 \%$, sendo Alcantarea nahoumii e Vriesea bahiana as plantas com maiores percentuais encontrados (Fig. 2). As espécies Alcantarea nahoumii e Vriesea bahiana pertencem à mesma subfamília Tillandsioideae, são rupícolas, cresciam em afloramentos rochosos, com ocorrência próxima em ambiente iluminado. Essas plantas embora tenham dimensões diferentes, apresentam as mesmas características morfológicas em suas estruturas essenciais (Pereira et al. 2009) e talvez tais características sejam responsáveis pela maior similaridade dos fungos encontrados.

A similaridade entre os vegetais investigados no Morro da Redenção apresentou índices entre

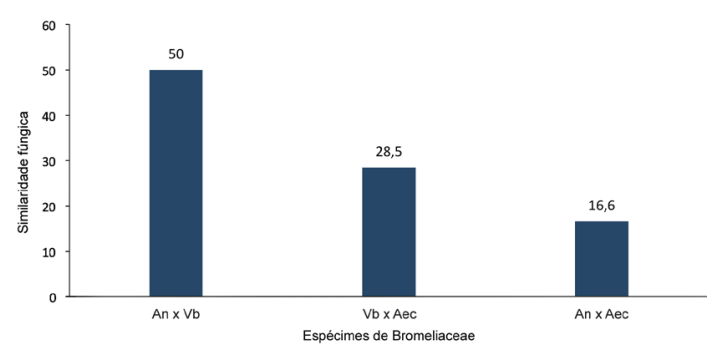

Figura 2 - Similaridade avaliada pelo índice de Sorensen entre populações de fungos ocorrentes em bromélias na Serra da Jibóia (Santa Terezinha), Bahia. $(\mathrm{An}=$ Alcantarea nahoumii $; \mathrm{Vb}=$ Vriesea bahiana ; Aec $=$ Aechmea $\mathrm{sp})$.

Figure 2 - Similarity evaluated through the Sorensen index among in populations of fungi occurring in bromeliads in the Serra da Jibóia (Santa Terezinha), Bahia. $(\mathrm{An}=$ Alcantarea nahoumii $; \mathrm{Vb}=$ Vriesea bahiana $;$ Aec $=$ Aechmea $\mathrm{sp})$. 
$33,3 \%$ a $58,8 \%$, ocorrendo maior registro para Aechmea aquilega e Hohenbergia catingae (Fig. 3). Estas espécies possuem estruturas foliares distintas, mas são rupícolas, pertencem à mesma subfamília Bromelioideae e estavam próximas no local de investigação. Os fungos comuns às duas espécies de bromélias são polífagos e a proximidade entre elas favorece a disseminação. Aechmea aquilega e Hohenbergia catingae são endêmicas do Brasil (Maia et al. 2015).

As bromélias estudadas a partir de material proveniente da Orquilândia Tropical revelaram uma similaridade entre $23,5 \%$ e $58,3 \%$, sendo Aechmea victoriana e Neoregelia compacta as mais semelhantes (Fig. 4). Os espécimes coletados nessa área estavam próximos entre si e apesar das estruturas diferenciadas das folhas, essa proximidade pode ter facilitado a disseminação dos fungos entre elas, sendo os fungos registrados bastantes comuns e com ampla gama de hospedeiros. Estas espécies de bromélias pertencem a subfamília Bromelioideae são epífitas e endêmicas do Brasil (Maia et al. 2015).

A diferença nos valores de similaridades pode estar relacionada aos ambientes dos locais de coleta, à proximidade genética e morfologia foliar das espécies estudadas. Tais fatores já foram observadas por Magalhães et al. (2011) estudando fungos anamorfos na serapilheira de árvores da Mata Atlântica encontrando similaridade variando de $45,9 \%$ a $60,9 \%$ entre as espécies de árvores. Esta variação maior que a constatada neste trabalho foi atribuída à diferença entre substratos (plantas de famílias muito distintas). Dessa forma, confirma-

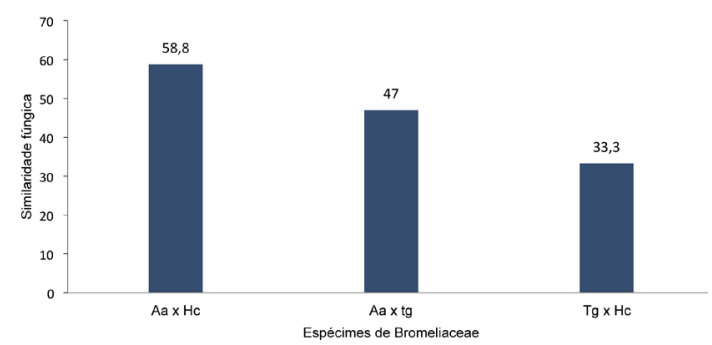

Figura 3 - Similaridade avaliada pelo índice de Sorensen entre populações de fungos ocorrentes em bromélias no Morro da Redenção (Campo Formoso), Bahia. (Aa = Aechmea aquilega; $\mathrm{Hc}=$ Hohenbergia catingae; $\mathrm{Tg}=$ Tillandsia gardneri).

Figure 3 - Similarity evaluated through the Sorensen index among in populations of fungi occurring in bromeliads in Morro da Redenção (Campo Formoso), Bahia. (Aa = Aechmea aquilega $; \mathrm{Hc}=$ Hohenbergia catingae; $\mathrm{Tg}=$ Tillandsia gardneri $)$.

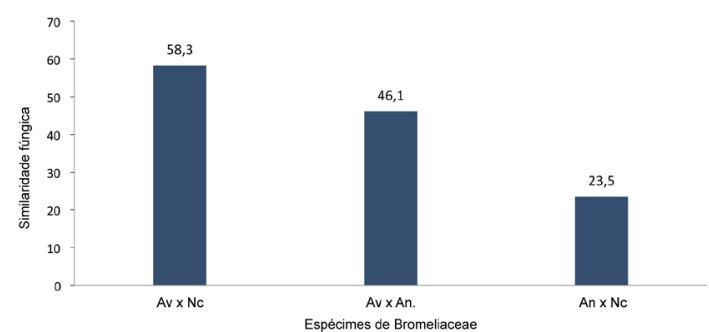

Figura 4 - Similaridade avaliada pelo índice de Sorensen entre populações de fungos ocorrentes em bromélias no Orquilândia Tropical (Camaçari), Bahia. $(\mathrm{Av}=$ Aechmea victoriana $; \mathrm{An}=$ Alcantarea nahoumii; $\mathrm{Nc}=$ Neoregelia compacta).

Figure 4 - Similarity evaluated through the Sorensen index among in populations of fungi occurring in bromeliads in Tropical Orquilândia (Camaçari), Bahia. (Av = Aechmea victoriana $; \mathrm{An}=$ Alcantarea nahoumii $; \mathrm{Nc}=$ Neoregelia compacta).

se a afirmativa de Polishook et al. (1996) de que amostras coletadas de uma mesma espécie de planta tendem a ter alta similaridade, comparadas às amostras de diferentes espécies vegetais em uma mesma área.

A partir dessa pesquisa, pioneira para Bromeliaceae, podemos constatar a importância do estudo da diversidade fúngica (micobiota) em bromélias, sendo as 27 espécies identificadas, um primeiro relato para esse vegetal e um suporte para outras pesquisas nessa área.

\section{Agradecimentos}

Os autores agradecem ao programa de Pós-graduação em Ciências Agrárias - UFRB e à Comissão de Aperfeiçoamento de Pessoal do Nível Superior (CAPES), a bolsa de Mestrado concedida a Patrícia M.G. Palha.

\section{Referências}

Araújo Filho JA \& Carvalho FC (1997) Desenvolvimento sustentado da Caatinga. Circular Técnico, 13. Embrapa-CNPC, Sobral. 19p.

Blengini IAD, Cintra MAMU, Cunha RPPC \& Caiafa AN (2015) Proposta de unidade de conservação da Serra da Jibóia, 2015. Disponível em <http:// www.gamba.org.br/wp-content/uploads/2016/03/ proposta-final.pdf $>$. Acesso em 10 outubro 2016.

Brower JE, Zar JH \& Von Ende CA (1998) Field and laboratory methods for general ecology. $4^{\mathrm{a}} \mathrm{ed}$. Wcb Mcgraw-hill, Boston. Pp. 273.

Brasil (2004) Ministério do meio ambiente. Secretaria de políticas para o desenvolvimento sustentável. Mata atlântica: o futuro é agora. MMA, Brasília. 16p. 
Castellani A (1967) A maintenance and cultivation of the common pathogenic fungi of man in sterile distilled water. Further researches. Journal of Tropical Medicine and Hygiene 70: 181-184.

Candeias EL, Santos MLC, Duarte EAA, Oliveira TAS, Bezerra JL \& Soares ACF (2016) Fungos endofíticos de raízes de sisal antagonistas ao Aspergillus. Agrotrópica 28: 29-36.

Costa AKF, Freire FCO, Vieira GP, Andrade JA \& Mendes FNP (2009) Fungi associated with Brazil nut and groundnut kernels sold in Fortaleza city (Brazil). Revista Ciência Agronômica 40: 455-460.

Costa IPMW, Assunção MMC, Lima TEF, Oliveira RJV \& Cavalcanti MAQ (2012) Checklist of endophytic fungi from tropical regions. Mycotaxon 119: 494.

Costa LA \& Gusmão LFP (2016) Communities of saprobic fungi on leaf litter of Vismia guianensis in remnants of the brazilian atlantic forest. Journal of Forestry Research 28: 1-10.

Dajoz R (1983) Ecologia geral. Ed. Vozes, Petrópolis. Pp. 472.

Ferreira JO (2010) Campo Formoso. Disponível em $<$ http://ambientalcampoformoso.blogspot.com.br/>. Acesso em 14 novembro 2016.

Ferreira JO (2013) Zonas fitogeográficas de Campo Formoso. Disponível em <http:// ambientalcampoformoso.blogspot.com. br/2013_06_01_archive.html>. Acesso em 03 junho $20 \overline{1} 6$.

Islair P, Carvalho KS, Ferreira FC \& Zina J (2015) Bromeliads in caatinga: an oasis for invertebrates. Biotemas, 28: 67-77.

Jensen CAS, Nascimento FC, Jensen RC, Rotondaro EE \& Saito RT (2011) Bromélia: promoção da diversidade $\times$ riscos á saúde ambiental. Revista Brasileira de Ciências Ambiental 19: 8.

Kumar P, Kandan A, Akhtar J \& Gawade BH (2015) Nigrospora oryzae: na endophyte of potential aplication in agriculture. Disponível em <http:// www.biotecharticles.com/agriculture-article/ nigrospora-oryzae-an-endophyte-of-potentialapplication-in-agriculture-3425.html>. Acesso em 1 dezembro 2016.

Lima TEF \& Cavalcanti MS (2014) Fungos endófitos e do filoplano de Caesalpinia echinata Lam da Estação Ecológica de Tapacurá, PE. Agrotrópica 26: 43-50.

Lima TEF, Oliveira RJV, Bezerra JL \& Cavalcanti MAQ (2014) Endophytic fungi from leaves and roots of Vitis labrusca cv. Isabel in Pernambuco/Brazil. Sydowia 66: 1-15.

Loiola MIB, Roque AA \& Oliveira ACP (2012) Caatinga: vegetação do semiárido brasileiro. Ecologia 4: 14-19.

Maia LC, Carvalho Júnior AA, Cavalcanti LH, Gugliotta AM, Drechsler ERS, Santiago ALM, Cárceres MES, Gibertoni TB, Aptroot A, Giachini AJ, Soares AMS, Seilva ACG, Magnago AC, Goto BT, Lira CRS, Montoya CAS, Zottarelli CLAP, Silva DKA, Soares DJ, Rezende DHC, Luz EDMN, Gumboski
ELWF, Karstedt F, Freire FM, Coutinho FP, Melo GSN, Sotão HMP, Baseia IG, Pereira J, Oliveira JJS, Sousa JF, Bezerra JL, Araújo Neta LS, Pfenning LH, Gusmão LFP, Neves MA, Capelari M, Melissa CWJ, Pelgarín MP, Júnior Menolli N, Medeiros PS, Friedrich RCS, Chikowski RS, Pires RM, Melo RF, Silveira MB, Urrea Valencia S, Cortez VG \& Silva VF (2015) Diversity of Brazilian fungi. Rodriguésia 66: 1033-1045.

Magalhães DMA, Luz EDMN, Magalhães AF, SantosFilho LP, Loguercio LL \& Bezerra JL (2011) Riqueza de fungos anamorfos na serapilheira de Manilkara maxima, Parinari alvimii e Harleyodendron unifoliolatum na mata atlântica do sul da Bahia. Acta Botanica Brasilica 25: 899-907.

Marques MFO, Gusmão LFP \& Maia LC (2008) Riqueza de espécies fungos conidiais em duas áreas de mata atlântica no Morro da Pioneira, Serra da Jibóia, BA, Brasil. Acta Botanica Brasílica 22: 954-961.

Muller-Dombois D (1981) Ecological measurements and microbial populations. In: Wicklow DT \& Carroll GC (eds.). The fungal community: its organization and role in the ecosystem. Marcel Derker, New York. Pp. 173-184.

Mussi-Dias V, Araújo ACO, Silveira SF, Rocabado JMA \& Araújo KL (2012) Fungos endofíticos associados a plantas medicinais. Revista Brasileira de Plantas Medicinais 14: 261-266.

Pereira AR, Andrade ACS, Pereira TS, Forzza RC \& Rodrigues AS (2009) Comportamento germinativo de espécies epífitas e rupícolas de Bromeliaceae do Parque Estadual do Ibitipoca, Minas Gerais, Brasil. Revista Brasileira de Botânica 32: 827-838.

Picanço MC (2010) Manejo integrado de pragas. Viçosa, MG. Departamento de Biologia Animal, Universidade Federal de Viçosa, Viçosa. 146p.

Polishook JD, Bills GF \& Lodge DJ (1996) Microfungi from decaying leaves of two rain forest trees in Puerto Rico. Journal of Industrial Microbiology 17: 284-294.

Queiroz LP, Sena TSN \& Costa MJSL (1996) Flora vascular da Serra da Jibóia, Santa Terezinha, Bahia. Vol. I: o campo rupestre. Sitientibus 15: 27-40.

Reis A, Boiteux LS \& Henz GP (2009) Antracnose em hortaliças da família Solanacea. Embrapa hortaliças, Comunicado técnico, Brasília. 9p.

Santos POD, Silva ACMD, Corrêa ÉB, Magalhães VC \& Souza JTD (2014) Additional species of Aspergillus causing bole rot disease in Agave Sisalana. Tropical Plant Pathology 39: 331-334.

Silva SS, Isabel TSS \& Gusmão LFP (2014) Fungos conidiais associados a substratos vegetais submersos em algumas áreas do bioma Caatinga. Rodriguésia 65: 527-538.

Silva VI \& Scatena VL (2011) Anatomia foliar de espécies de Bromeliaceae (Poales) da Amazônia, Mato Grosso, Brasil. Revista de Ciências AgroAmbientais 9: 225 - 240. 
SOS Mata atlântica - INPE (2013) Atlas dos remanescentes florestais da Mata Atlântica, período 2011 - 2012, São Paulo. Disponível em <https://www.sosma.org.br/wp-content/ uploads/2013/06/atlas_2011-2012_relatorio_ tecnico 2013final.pdf>. Acesso em abril 2017.

Souza EH, Souza FVD, Rossi ML, Brancalleão N, Ledo CAS \& Martinelli AP (2015) Viability, storage and ultrastructure analysis of Aechmea bicolor (Bromeliaceae) pollen grains, an endemic species to the atlantic forest. Euphytica 204: 13-28.

Tokeshi H (2002) Doenças e pragas agrícolas geradas e multiplicadas pelos agrotóxicos. Revista cultivar grandes culturas, $\mathrm{n}^{\circ}$. 39. Disponível em $<$ http:// www.grupocultivar.com.br/artigos/doencas-epragas-agricolas-geradas-e-multiplicadas-pelosagrotoxicos>. Acesso em 16 novembro 2016. 